\title{
Maxillary sinus augmentation - diagnostic and surgical technique
}

\author{
Anca Delia Vereanu ${ }^{1}$, Dana Tomescu², Manuela Andra Savu ${ }^{3}$, Codruț Sarafoleanu ${ }^{3}$
}

${ }^{1}$ Departament of Oral Implantology, Clinical Hospital of Oro-Maxillo-Facial Surgery

"Prof. D. Theodorescu", UMF Carol Davila, Bucharest, Romania

'Department of Anesthesiology and Intensive Care III, "Fundeni" Clinical Institute,

Bucharest Romania

${ }^{3}$ ENT\&HNS Department, "Sfanta Maria" Hospital, Bucharest, Romania

\section{ABSTRACT}

Placing dental implants in edentulous patients is a difficult task, as the anatomy of patients has a high variability. The expansion of the pneumatized maxillary sinus after tooth extraction is the biggest impediment of all. Because of the undersized ridge width patients need bone grafting in order to compensate the loss of their own. This procedure is performed by the oro-maxillo-facial surgeon who evaluates, advises and offers the patient the best medical guidance. A series of surgical techniques and surgical implants have been performed in the field of bone grafting, but only a few of them are now being used.

Key words: dental implant, sinus lift, maxillary sinus, surgery

\section{INTRODUCTION}

The maxillary sinus is a cavity, an aerated space that occupies the maxillary bone and its elongations through inconstant recesses. (1) The shape and form of the maxillary sinus resemble an overturned pyramid: the base is the intersinusonasal wall and the apex is towards the zygomatic bone. The air volume inside the sinus depends of the pneumatization of the sinus, having approximately $15 \mathrm{ml}$ air space. (2-4) It is the only sinus that has a non-physiologic, antigravity drainage. The floor of the maxillary sinus is formed by the alveolar recess; in adults the sinus floor is situated below the nasal level. (figure 1)

The anterior wall presents the canine fossa, the thinnest maxillary bone region, the place to access towards the sinus cavity. Mainly, the maxillary is a finely trabecular bone, with a lower density than the mandible. This consideration is important as the medullary bone must establish a stress-bearing surface for the implant in order to create a functional system; the implant must remain fixed and, in the same time, be able to transmit the mastication forces to the supporting bone. $(5,6)$ Placing dental implants, after sinus lift interventions, is the best procedure to recreate a physiological dentition for patients with atrophic posterior maxilla. (7) (figure 2)
Corresponding author: Dana Tomescu MD, PhD Department of Anaesthesia and Critical Care III, Fundeni Clinical Institute Associate Professor "Carol Davila" University of Medicine and Pharmacy 258 Fundeni street, Bucharest, Romania E-mail: danatomescu@gmail.com 


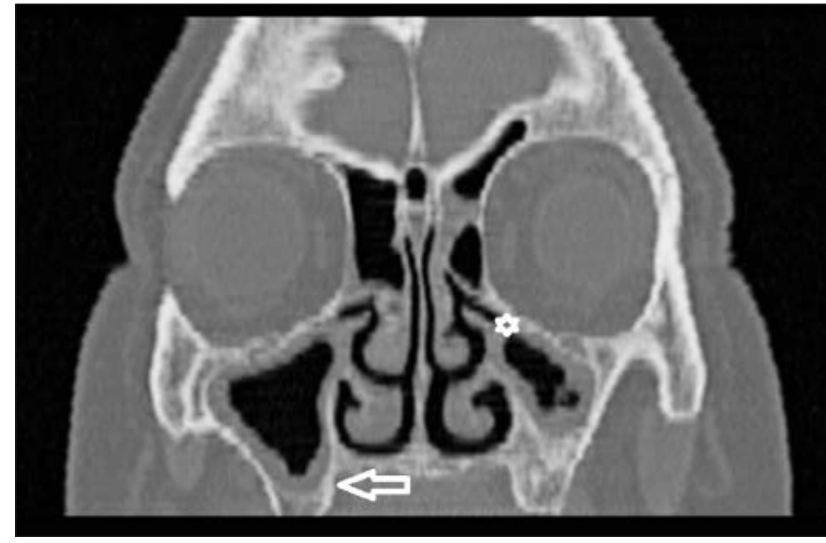

Figure 1 - Cranio-facial CT scan (coronal sections): left maxillary sinus with secretions - the star marks the drainage ostium

(antigravitational drainage); the arrow points the right maxillary sinus with the lowered floor and a thick endosinusal mucosa

(personal archive)

The improper ridge width, caused by the hyperpneumatization of the sinus (figure 3 ), create difficult situation for the implantologist. (8) Sometimes the distance from the maxillary floor to the alveolar ridge is $1 \mathrm{~mm}$ thin. (9)

Tatum was the first to describe the grafting of the antral floor. $(5,10,11)$ He developed and described the procedure. For the first time he used the alveolar crest access to the maxillary sinus. Afterwards, a modified Caldwell-Luc access was used, piercing the lateral wall of the maxilla and elevating the Schneiderian membrane. In the years to come other authors published various techniques, multitudinous types of implant were used, as well as different sites of material grafting: in 1980 Boyne placed autogenous marrow and cancellous bone inside the sinus (12), in 1984 Misch used a blade-vent implant (13) and in 1997 Garg modified the shape of the bone window (14).

There are to possible approach ways for sinus lift grafting and implant placement: one or two-step procedure; both of them have been reported with successful outcomes. (15-18) For the first option (the one-step procedure) the alveolar bone needs to have a proper width (more than $5 \mathrm{~mm}$ ), the sinus must be partial pneumatized, in order to execute bone grafting and implant insertion in the same intervention - the bone graft will consolidate around the implant (8).

If the alveolar bone is less than $5 \mathrm{~mm}$ the two-step procedure is desirable. The surgeon will execute the sinus floor elevation and, after 4 to 6 months, the dental implants will be inserted. $(16,17)$ Other authors consider that $1 \mathrm{~mm}$ height of the floor is sufficient for the one-step intervention. (19-22)

Prior to surgery the patient must be accurately

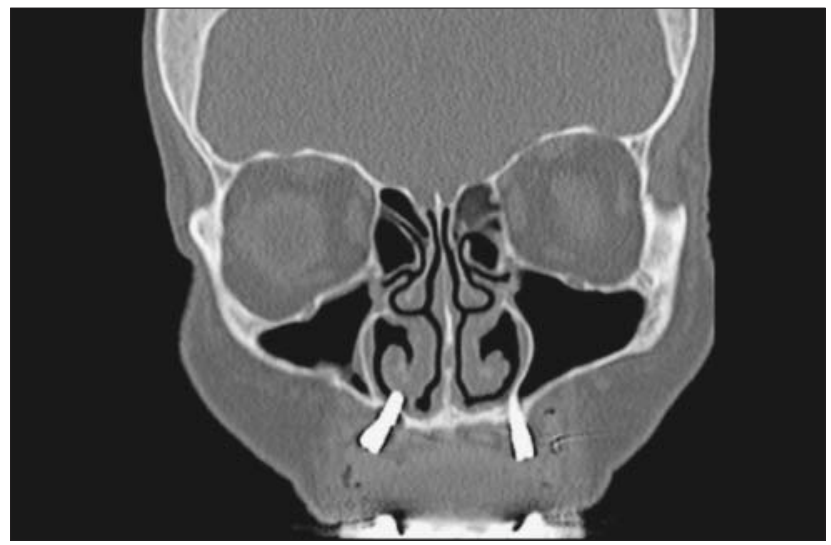

Figure 2 - If there is no consideration for the local anatomy the outcome of the dental implant is poor and the physiologic result is unfortunate. (personal archive)

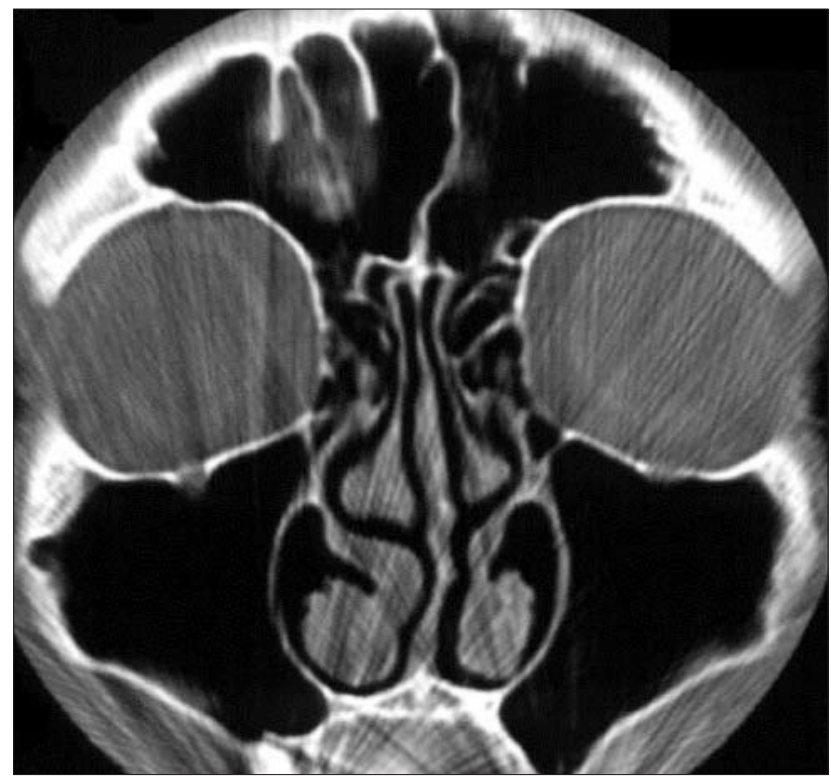

Figure 3 - Example of a pathologic state of pneumatization - all situations should be thoroughly explored prior to surgery (personal archive)

evaluated, including medical history and habits [e.g. use of tobacco - as nicotine diminishes tissue healing and inhibits osteoblast activity (23)] A patient with otorhinolaryngological diseases must be first treated by the ENT specialist.

Imaging of the maxillary sinus is imperative: OPG (orthopantomography) and even sinus radiography or a CT sinus scan - it will provide to the surgeon the anatomic status: the alveolar bone height, the pneumatization intensity, the location of the sinus floor, arterial conformation (figure 4), and the possible endosinusal mucosa pathology (polyps, tumors, cysts, sinusitis, foreign bodies). 


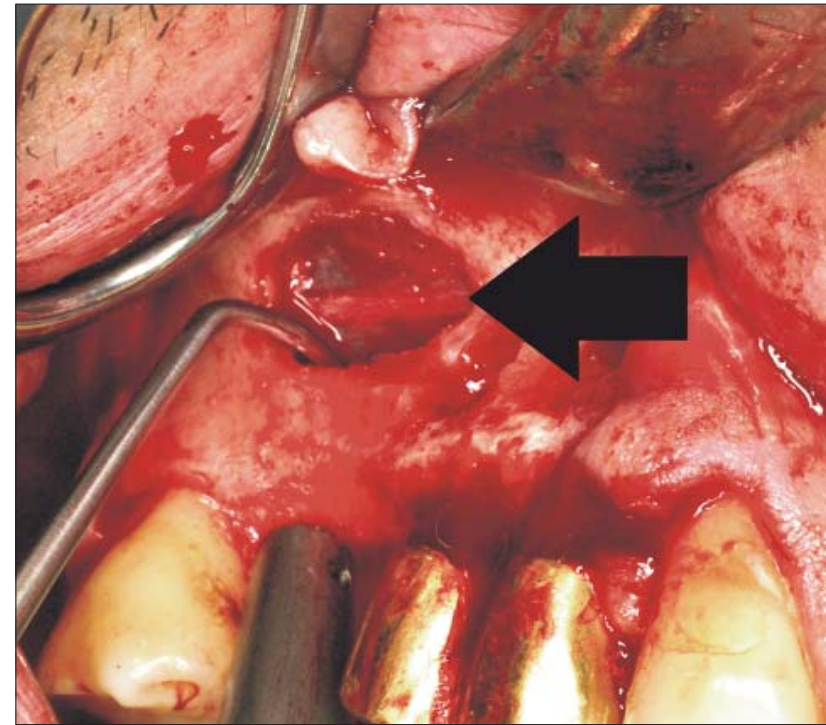

Figure 4 - After drilling the anterior maxillary wall an arterial structure is encountered. This situation is to be foreseen on a CT scan. (personal archive)

\section{Assessing the maxillary sinus}

When the imagistic examinations achieved are abnormal, the oro-maxillo-facial doctor can refer the patient to an ENT surgeon. After the clinical ENT examination and the CT-scan interpretation he can recommend a sinusoscopy - a mini-invasive surgical procedure that allows direct visualization of the sinus cavity. It offers a panoramic view, different from the direct normal view. (1) Aside from the surgical manoeuvres (removing: foreign bodies, fungoid material, benign tumors as cyst, polyp, mucocele) sinusoscopy is a great method to evaluate the sinusal mucosa, furnishing valuable data to the implantologist postponing or not the intervention. Inside the maxillary sinus there can be five types of mucosa transformation stages, as Sarafoleanu published his classification: (1)

Type 0 - normal mucosa and vascular pattern, sinus without secretions; the mucolilliary and ostial function are normal.

Type I - pronounced vascular marking, a little bit "dull", with a few serous secretions.

Type II - thick mucosa, substantial secretions, marked vascular design, dilated glandular ducts, corium alteration. In this stage, this modification responds to medication or to minimal surgical procedures directed to ostial permeabilisation.

Type III - intense modification of the mucosa: polyps, cysts, mucopurulent secretions. Only endoscopic sinus surgery will help. (figure 5)

Type IV - hyperplasic and metaplasic mucosa, organised polyps, fungosities and caseum. In this case

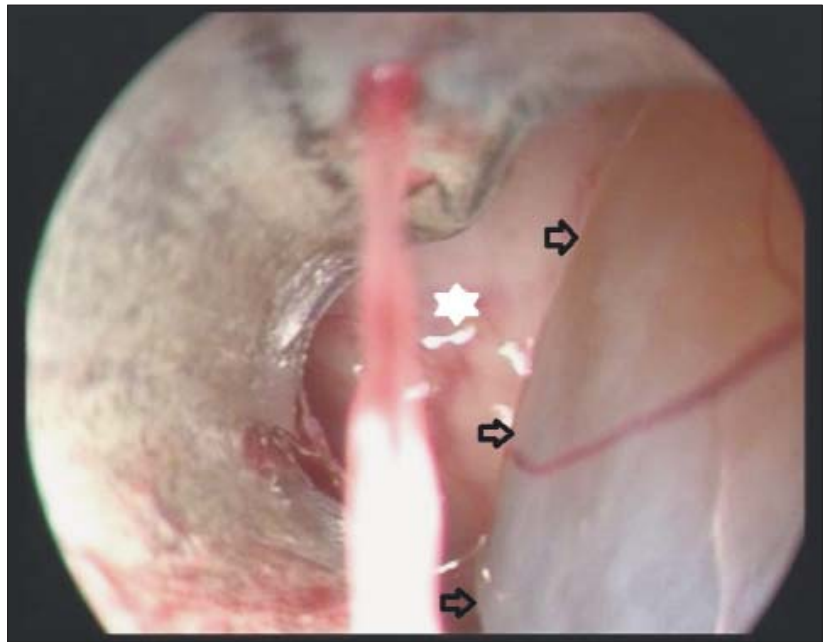

Figure 5 - Endoscopic view of the maxillary sinus. The black arrows are pointing the margins of a large cyst that will be removed through sinusoscopy. The white mark shows the endosinusal mucosa with visible vascular pattern. (personal archive)

the endoscopic surgery has a poor outcome; often radical classic surgery (Caldwell-Luc technique) is indicated.

Whenever the ENT surgeon considers, tissue fragments can be harvested and sent to a histopathologic examination - the anatomopathologist doctor will provide additional data regarding the sinusal mucosa, announcing the surgical outcome of the dental implant intervention.

\section{Materials used for grafting}

Bone grafting, or transplanted osteogenesis, is a dynamic process not a solid bone block that integrates itself into place. (24) It involves cellular regeneration and the mineralisation of the osteoid: osteogenesis, osteoinduction and/or osteoconduction. (25-28)

Autogenic bone graft are used most often (15$17,29,30)$ - the iliac crest is most accessed harvesting site. $(15,30)$

Until now, there is no universal agreement as to which material (or combination of) is the best to use to elevate the maxillary floor.

Other materials were used, like: bone allografts $(25$, $28,31)$, TCP (tricalcicum phosphate), resorbable and nonresorbable hydroxyapatite $(8,28,32-34)$, bovinederived bone mineral (35) and bioactive glasses. (36)

The ideal maxillary sinus bone-grafting material should provide biologic stability, ensure volume maintenance, and allow the occurrence of new bone infiltration and bone remodeling. (37) The ideal graft should be a strong nontoxic, nonantigenic, noncarcinogenic matherial, easy to fabricate, resistant to 
inflamation and infections, to permit tissue adhesion and low-priced. (38)

\section{SURGICAL TECHNIQUE}

\section{Anesthesia}

The surgical intervention can be performed under local anesthesia alone or, in some cases, patient can receive intravenous analgosedation or general anesthesia, provided by the anesthesiologist and with standard monitoring. The preoperative evaluation has to be performed. An informed consent has to be signed by the patient explaining additional risks due to anesthesia.

In the local anesthesia technique the substance with vasoconstrictor (e.g. Lidocaine clorhidrat injectable solution $1 \%$ with epinephrine 1:100.000/ 1:200.000 or mepivacaine or bupivacaine) is infiltrated into the operatory field and in the sites of donor grafting regions. Postero-superior alveolar nerve and the greater palatine nerve will be also blocked. (36)

Analgosedation provides anxiolysis and analgesia but the patient is responsive and breathes spontaneously, avoiding postoperative complications. It is a safe approach.

General anesthesia is rarely needed, mainly in extensive procedures, anxious patients and in children. The need for grafting from the hip, simultaneous placement of multiple implants require general anesthesia. A protocolized approach regarding discharge after general anesthesia has to be followed, based upon standardized criteria.

\section{Accessing the sinus}

Before making any incision a series of factors must be evaluated, as: the position of the sinus floor, the gingival status, the presence of healthy teeth and the position of the succeeding antrostomy.

The first incision is made on the alveolar crest (rarely on the palatal side - recommended in case of muscular insertion on the ridge): a horizontal, deep cut, extended anterior beyond the sinus limit. The second incision is a vertical one, up to the vestibule, releasing the flap and exposing the bone. Next step is elevation of the periosteum - minimal striping is required in order to preserve the alveolar vascularisation. (figure 6)

The lateral wall is now exposed - with a burr drill a window is created. To avoid the injury of the Schneiderian membrane, when approaching it, a diamond drill should be used. Many osteotomy techniques were described - the oval shape is recommended as the sharp edges can perforate the membrane. (14)

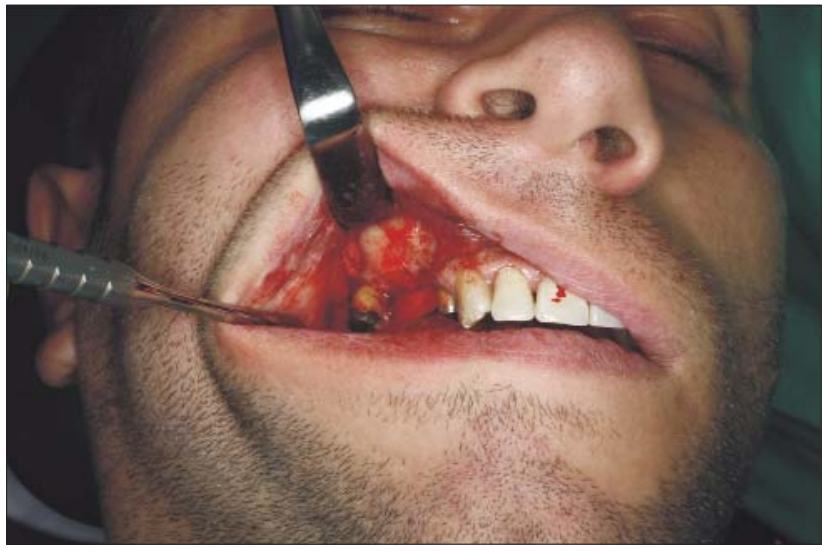

Figure 6 - After the mucoperiostal flap is elevated

the alveolar ridge and the anterior maxillary wall are exposed. (personal archive)

(figure 7) The bony window created can be removed or used as a roof of the graft. (figure 8) With a curete the Schneideriam membrane is circumferentially separated from the bony structure, carefully, without tearing it. (figure 9) The most common aereas of perforation are at the level of inferior osteotomy and the inferomedial portion of the sinus window (8) - small perforation can be managed with collagen material. A large perforation is handled using a large collagen membrane, placing it as a cover between the Schneiderian membrane and the grafted bone.

After membrane perforation, the second most frequent complication encountered is bleeding. Most of the times the bleeding happens from the soft tissue or form the bony capillaries, frequently correlated with the patient's bood pressure. In extreme, the intervention must be stopped if the hypertension can't be

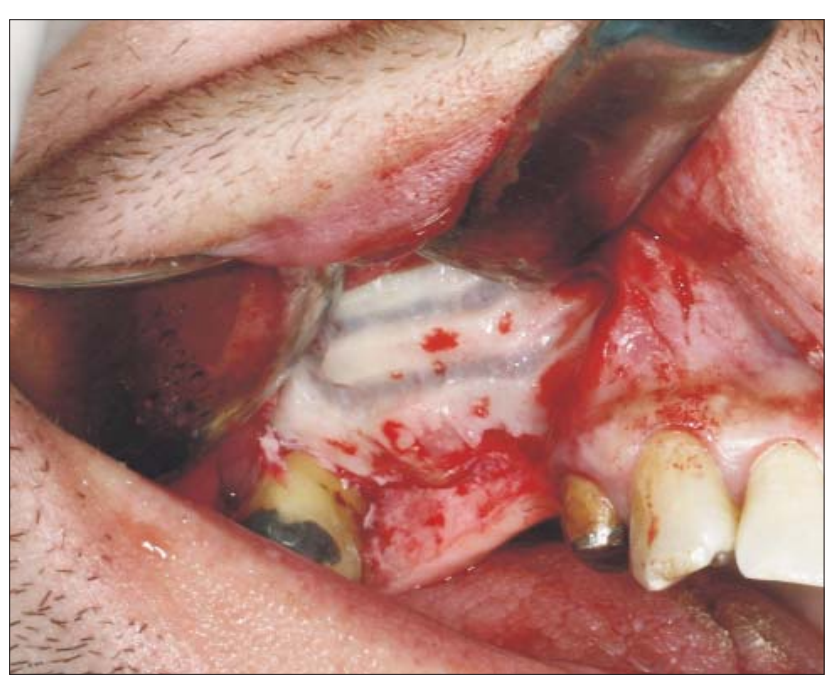

Figure 7 - The maxillary (right) window is circumferentially drilled. (personal archive) 


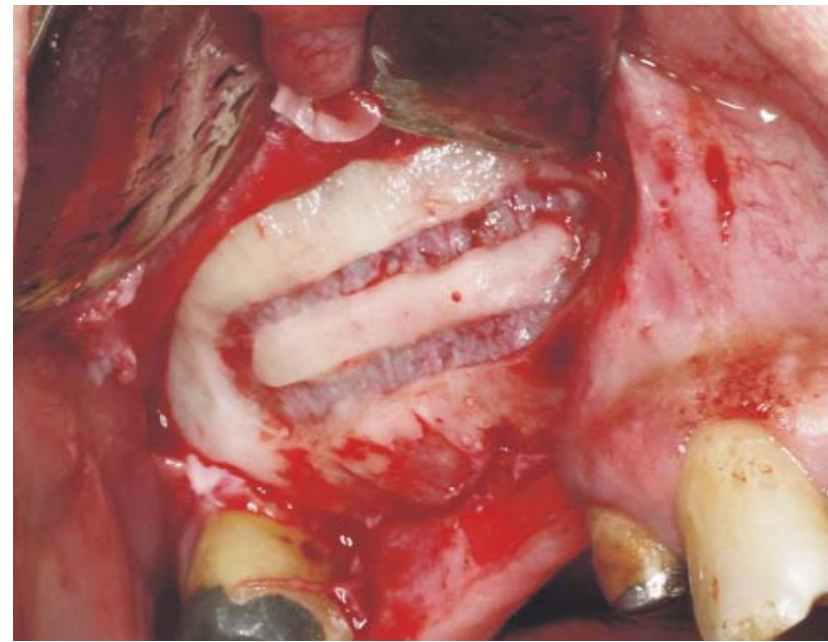

Figure 8 - The bony window is attached only with the Schneiderian membrane. (personal archive)

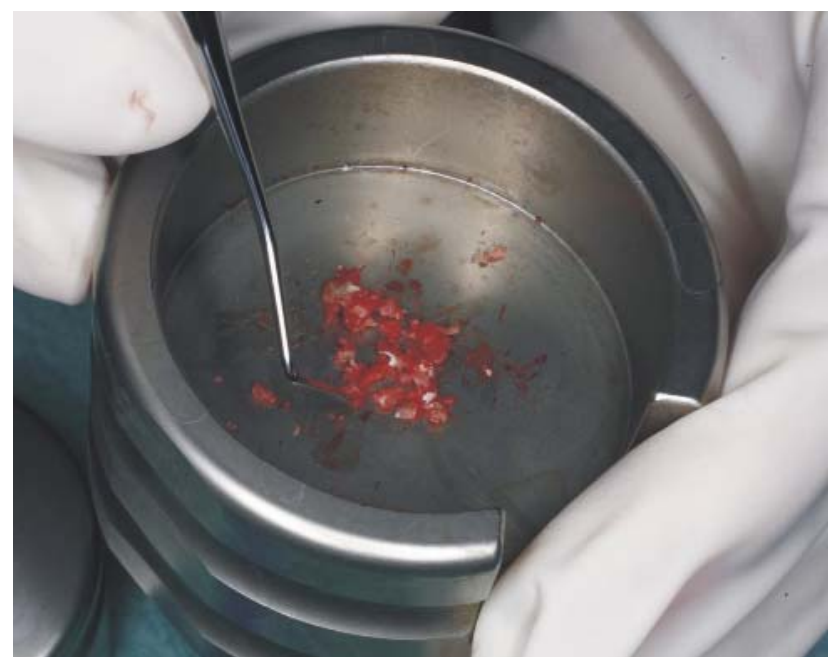

Figure 10 - Triturating the bone removed from the anterior wall. (personal archive)

controled by adjusting the anesthesia or/and using supplematary sedation.

\section{Placing the graft}

As mentioned before, the grafting material is cropped from selected areas; we use the bony window too, after triturating it. (figure 10) This autologue graft can be mixed with other materials, in order to improve its proprieties. (figure 11)

In the case of the one-step surgery (when the implant and the graft are placed in the same intervention) supplementary clinical measurements will be necessary to ensure the implant parallelism. (19) (figure 12) After the implants are placed, bone graft is introduced and pressed to the maxillary walls, covering the implant(s). (figure 13) The access window is closed

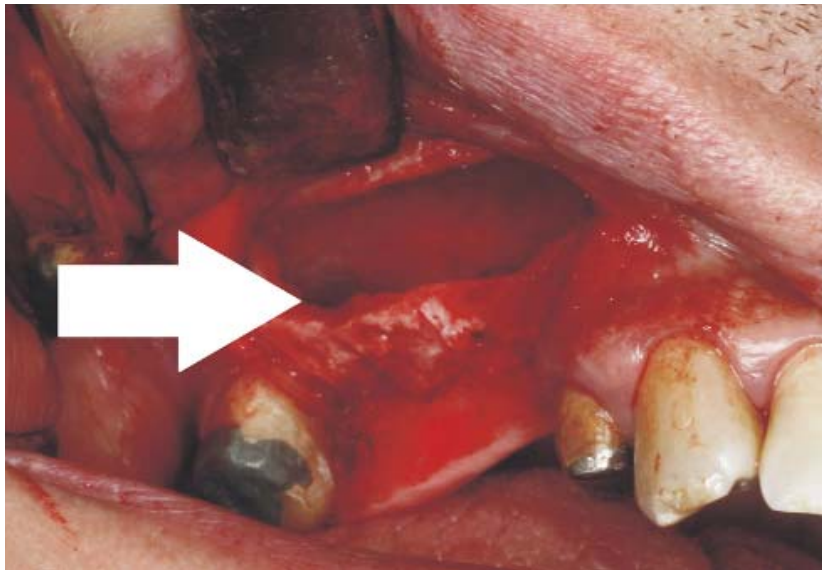

Figure 9 - After carefully creating the window and removing the bone the Schneiderian membrane is elevated from the sinus floor. (personal archive)

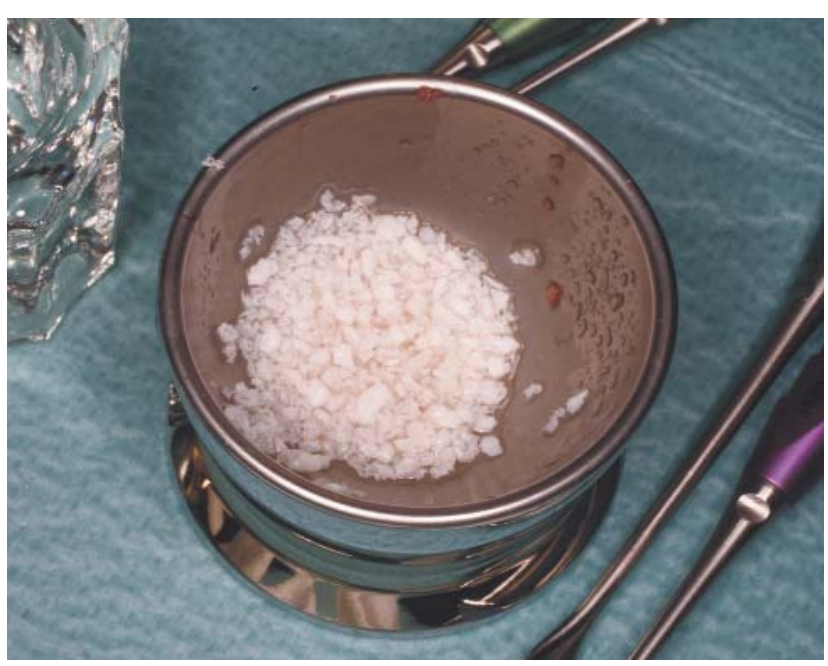

Figure 11 - The final graft mix. (personal archive)

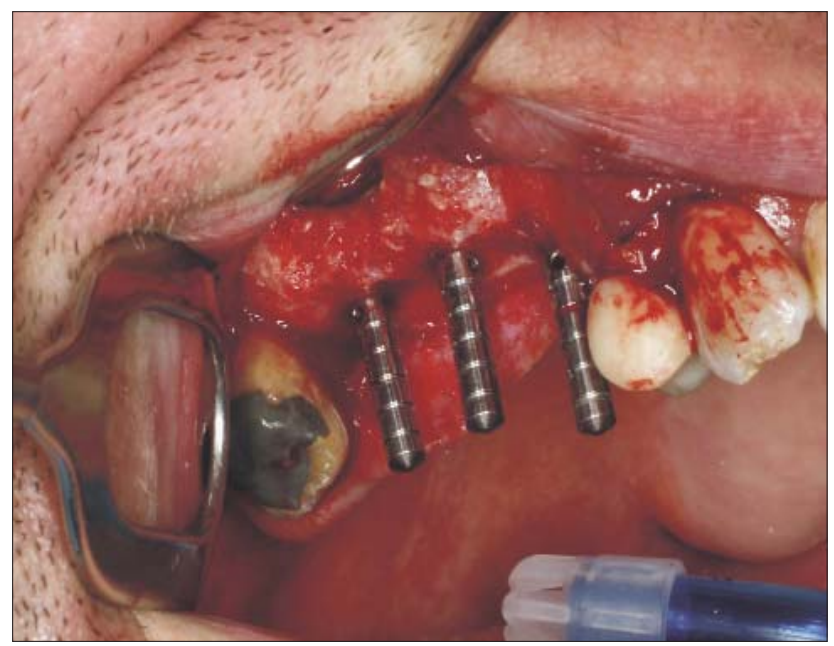

Figure 12 - Assuring the implant parallelism. (personal archive) 


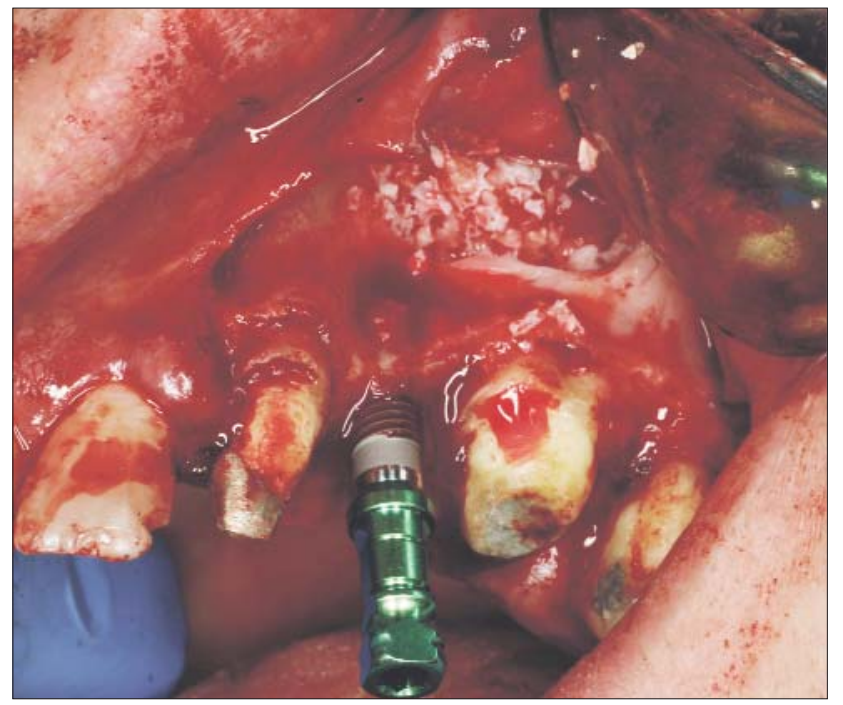

Figure 13 - The one-step procedure: the graft is placed between the sinus floor and the Scheniderain membrane. The dental implant is being screwed in. (personal archive)

by placing a barrier, a membrane to close the maxillary sinus. The mucosa flap is replaced and the incision is fixed with sutures.

A two-step procedure is performed using the same succession of surgical steps, up to the dental implant this step will be excecuted in the second stage, after 4 to 12 months. The material graft is placed and the opening is wrapped with a resorbable material and then the mucoperiosteal flap is sutured.

\section{CONCLUSIONS}

As simple as may seem, dental implant surgery is a challenging experience. A standardized surgical technique has not been yet universally approved. Every patient must be accurate assessed, especially by imagistic examinations - anatomical measurements will provide the surgeon all the basic information in order to establish the correct treatment plan. If the bone ridge has a right width, the one-step surgery is preferable. Any experienced surgeon will know to manage the complications (bleeding, membrane perforation) and to offer the best surgical outcome.

\section{REFERENCES}

1. Sarafoleanu C. Rinologie. Editura Medicala. Bucuresti. 2003

2. Chanavaz M. Maxillary sinus: Anatomy, physiology, surgery, and bone grafting related to implantology - Eleven years of surgical experience (1979-1990), J Oral Implantol. 1990;16:199-209.

3. Cuenin MF, Pollard BK, Elrod CW. Maxillary sinus morphology in differential dental diagnosis. Gen Dent. 1996;44(4):328-331.

4. Ulm CW, Solar P, Gsellman B, Matejka M, Watzek G. The edentulous maxillary alveolar process in the region of the maxillary sinus -A study of physical dimension. Int J Oral Maxillofac Surg 1995;24:279-282.

5. Tatum H Jr. Maxillary and sinus implant reconstructions. Dent Clin North Am 1986;30:207-229.

6. Razavi R, Zena RB, Khan Z, Gould AR. Anatomic site evaluation of edentu- lous maxillae for dental implant placement. J Prosthodont 1995;4:90-94.

7. Chanavaz M. Sinus grafting related to implantology. Statistical analysis of 15 years of surgical experience (1979-1994). J Oral Implantol 1996;22:119130

8. Smiler DG. Johnson PW. Lozada JL. et al: Sinus lift grafts an endosseous implants: Treatment of the atrophic posterior maxilla. Dent Clin North Am 1992;36: 151-186.

9. Khoury F. Augmentation of the sinus floor with mandibular bone block and simultaneous implantation: A 6-year clinical investigation. Int J Oral Maxillofac Implants 1999;14:557-564.

10. Tatum H Jr. Endosteal implants. CDA J 1988;16:71-76

11. Tatum H. Maxillary implants. Florida Dent J 1989;60:23-27.

12. Boyne PJ, James RA. Grafting of the maxillary sinus floor with autogenous marrow and bone. J Oral Surg. 1980;38:613-616.

13. Misch CE. Maxillary sinus augmentation for endosteal implants: organized alternative treatment plans. Int J Oral Implant 1987;4:49-58

14. Garg AK, Quipones CR. Augmentation of the maxillary sinus: a surgical technique. Pract Periodontics Aesthet Dent. 1997:9:211-219.

15. Kent JN, Block MS. Simultaneous maxillary sinus floor bone grafting and placement of hydroxylapatite-coated implants. J Oral Maxillofac Surg. 1989; $47: 238-242$.

16. Jensen $J$, Simonsen EK, Sindet-Pedersen S. Reconstruction of the severely resorbed maxilla with bone grafting and osseointegrated implants: A preliminary report. J Oral Maxillofac Surg. 1990;48:27-32.

17. Raghoebar GM, Brouwer TJ, Reintsema H, Van Oort RP. Augmentation of the maxillary sinus floor with autogenous bone for the placement of endosseous implants: A preliminary report. J Oral Maxillofac Surg. 1993; 51:1198-1203.

18. Zinner ID, Small SA. Sinus lift graft: Using the maxillary sinus to support implants. J Am Dent Assoc 1996;127:51-57.

19. Peleg M, Mazor Z, Chaushu G, Gark A: Sinus Floor Augmentation with Simultaneous Implant Placement in the Severely Atrophic Maxilla. J Periodontol,1998.69; 1397-1403

20. Peleg M, Mazor Z, garg AK. Augmentation grafting of the maxillary sinus and simultaneous implant placement in severly atrophic maxilla. J Periodontol 1998:69:1937-1403

21. Boyne PJ. The use of Bone Graft Systems in Maxillary Implant Surgery. (Proceedings of the 50th Annual meeting of the American Institute of Oral Biology. 29 Oct-3 Nov 1993, Palm Springs, CA.) 1994;107-114

22. Daelemans $P$, Hermans $M$, Godet $F$, and Malevez $C$. Autologous bone graft to augment the maxillary sinus in conjunction with immediate endosseous implants: a retrospective study up to 5 years, Int J Periodontics Restorative Dentistry, 1997;17;27-39.

23. Hollinger JO, Schmitt JM, Hwang K, Soleymani P, Buck D. Impact of nicotine on bone healing. J Biomed Mater Res 1999;45:294-301(erratum 1999:46:438-439).

24. Marx, RE, Garg AK. Bone structure, metabolism, and physiology: Its impact on dental implantology, Implant Dent 1998;7:267-276.

25. Lane JM. Bone graft substitutes. West J Med 1995;163;565-566.

26. Frame JW. Hydroxyapatite as a biomaterial for alveolar ridge augmentation. Int J Oral maxillofac Surg 1987:16:642-655.

27. Pinholt EM, Bang G, Haanaes HR. Alveolar ridge augmentation in rats by combined hyroxyapatite and osteoinductive material. Scand J Dent Res 1991;99;64-74.

28. Misch CE, Dietsh F. Bone-grafting materials in implant dentistry. Implant Dent 1993:2:158-167.

29. Blomqvist JE, Alberius $P$, Isaksson $S$, Linde A, Obrant K. Importance of bone graft quality for implant integration after maxillary sinus reconstruction. Oral Surg Oral Med Oral Pathol Oral Radiol Endod. 1998:86:268-74.

30. Jensen J Sindet-Pedersen S. Autogenous mandibular bone grafts and osseointegrated implants for reconstruction of the severely atrophied maxilla: a preliminary report. J Oral Maxillofac Surg. 1991;49:1277-87.

31. Wood RM, Moore DL. Grafting of the maxillary sinus with intraorally harvested autogenous bone prior to implant placement. Int J Oral Maxillofac Implants 1988:3:209-214

32. Fetner AE, Hartigan MS, Low SB. Periodontal repair using PerioGlas in nonhuman primates: Clinical and histologic observations. Compendium 1994; 15:932,935-938.

33. Schepers E, de Clercq M, Ducheyne P, Kempeneers R. Bioactive glass particulate materials as filler for bone lesions. J Oral Rehabil 1991:18:439-452

34. Schepers EJ, Ducheyne P, Barbier L, Schepers S. Bioactive glass particles for narrow size range: A new material for the repair ofbone defects. Implant Dent 1993:2:151-156.

35. McAllister BS, Margolin MD, Cogan AG, Buck D, Hollinger JO, Lynch SE. Eighteen-month radiographic and histologic evaluation of sinus grafting with anorganic bovine bone in the chimpanzee. Int $J$ Oral Maxillofac Implants 1999;14:361-368.

36. Arun K Garg. Bone. Biology, Harvesting, Grafting for Dental Implants. Rationale and Clinical Applications. Quintessence Publishing. 2004. IL.

37. Pal US, Nanda Kishor Sharma, Singh RK, Shadab Mahammad, Divya Mehrota, Nimisha Singh, Davendra Mandhyan. Direct vs. indirect sinus lift procedure: A comparison. Natl J Maxillofac Surg. 2012 Jan-Jun; 3(1): 31-37.

38. Wagner J. Clinical and histological case study using resorbable hydroxylapatite for the repair of osseous defects prior to endosseous implant surgery. J Oral Implantol 1989;15:186-192. 